\section{Notes on the Application of Wet Ice}

\author{
By Dena Gardner, M.C.S.P.
}

The application of cold has proved an effective means of physical therapy. The physiological effect of cold applied for a short duration is remarkably similar to that of heat; it appears moreover that the effect of the former lasts longer and is a more suitable preparation for activity because the patient feels refreshed, as for example, after a cold bath or shower, in contrast to the feeling of ennervation which results from the application of heat. Normal functional activity is the ultimate aim of most physiotherapeutic procedures and cold applications help to accelerate the patient's physical rehabilitation.

\section{Equipment}

The requirements are simple and inexpensive, i.e. some medium-sized turkish towels and an ordinary household bucket three-quarters full of ice. Flaked or shaved ice is preferable because it is not lumpy and adheres more readily to the surface of the towels, however finely crushed ice is quite satisfactory and easier to obtain. Two wet and folded towels are placed between layers of ice in the bucket and water is added to a depth of about two or three inches in the bottom of the bucket. It is essential that the temperature of the ice and water mixture is as low as possible so that the flakes or fragments of ice adhere to the surface of the towels; ice cubes are unsuitable as they fail to reduce the temperature sufficiently and also make the application uneven and uncomfortable for the patient. Plinths upholstered in plastic

\section{(Continued from page 8)}

The obvious development is that once such a pilot scheme operates successfully, it creates a demand for an augmented service which cannot reasonably be expected from voluntary workers. It will, of course, be realised, that not every establishment will require a daily, full-time, physiotherapy service. Part-time physiotherapists could be employed, or several institutions could pool their efforts and organise physiotherapy on alternating days.

Two Johannesburg institutions which started physiotherapy through pilot schemes, have indeed already introduced regular day sessions, fully integrated with the medical I services, so as to fulfil the increased demand for physiotherapy.

\section{Summary}

It is felt that at this early stage of the organisation of comprehensive geriatric services in our country, physiotherapy cannot afford to sit back and wait for invitations. In view of the great contribution that physiotherapy can make in the care of the aged, we should not be reluctant to come forward and offer our services. Our branches, and individual members, could be of great value to local establishments for the welfare of the aged, and should express their willingness to co-operate, advise, and assist as members of the tean.

They would thus enhance the efficiency and scope of existing services and ensure for physiotherapy the place it deserves in the care for the aged.

Where locally physiotherapy services already exist, and branches and members are participating in the care for the aged, the S.A.S.P. should receive detailed information. The C.E.C. will then be able not only to express appreciation of the contributions made by our members, but also to correlate the experience of all our workers, provide an exchange of views and increase the value of our effort. materials make the use of waterproof or plastic sheeting unnecessary, a spare towel is all that is required to dry the patient and to mop up after the application.

\section{Technique}

When the patient is adequately undressed and ready for treatment the therapist takes one folded towel from the ice bucket, rings it quickly and thoroughly, unfolds it to the required size and places it firmly on the patient's skin. As the towel is unfolded flakes of ice are seen to adhere to the towel, any delay or shaking of the towel must be avoided as this would raise the temperature and consequently reduces the effect of the application. Once the towel is in contact with the patient's skin its temperature rises rapidly, it must therefore be removed after two or three minutes and replaced by another which has been soaking in the bucket. Three or four towels are usually sufficient.

\section{Effects and Uses}

\section{The Relief of Pain}

Cold decreases the speed of nerve conduction therefore the feeling of pain is reduced. Ice towels are applied to the painful area as frequently as possible. Pain is a factor which limits movement and inhibits muscular contraction, it is therefore often possible to obtain an increased range of movement or a more satisfactory contraction of muscles while the ice towel is in situ.

\section{The Relief of Spasm}

Cold decreases the speed of nerve conduction and the stretch stimulus is inhibited. For the reduction of spasm the ice towel must cover the whole length of the affected muscles. Reduction of spasm in a whole limb is usually most satisfactory when cold is applied to the distal parts first. Subsequent treatments produce reduction in spasm in a shorter time and the effect is more prolonged.

\section{Active Hyperaemia}

Cold applications of short duration reduce the superficial blood supply initially but this is followed by an active hyperaemia which is apparent when the towels are removed. This local increase in the circulation is useful for the reduction of swelling and prior to joint mobilisation. Ice towels are applied over the required area, or a hand or foot may be put directly into the bucket, immersion of hands or feet must be of short duration initially however, as sensation is acute in these areas.

\section{Contra-indications}

There appears to be very few contra-indications to treatment with ice, most parients like it and find it more effective than heat. Patients with circulatory disorders such as Reyauds and Buergers disease are however unsuitable for this type of treatment.

\section{Advantages}

The use of wet ice as described has many advantages: $(a)$ it is effective in a wide variety of conditions, $(b)$ it is time-saving for both patient and therapist as many active and passive exercise techniques can be continued while the ice towels are in situ, $(c)$ applications of cold can safely be carried out at frequent intervals by the patient in his own home provided a refrigerator is available.

As in the case of any other technique of physical treatment, care and accuracy in the application is essential together with acute observation of the results obtained. 